Research Paper

\title{
Antibiotic Elution Characteristics and Pharmacokinetics of Gentamicin and Vancomycin from a Mineral Antibiotic Carrier: An in vivo Evaluation of 32 Clinical Cases
}

\author{
Thomas Colding-Rasmussen ${ }^{\bowtie}$, Peter Horstmann, Michael Mørk Petersen, Werner Hettwer \\ Department of Orthopaedic Surgery, the Musculoskeletal Tumour Section, Rigshospitalet, University of Copenhagen. \\ $\square$ Corresponding author: Thomas Colding-Rasmussen, the Musculoskeletal Tumour Section, Blegdamsvej 9, 2100 Copenhagen $\varnothing$, Email: \\ Thomascolding@gmail.com \\ (c) Ivyspring International Publisher. This is an open access article distributed under the terms of the Creative Commons Attribution (CC BY-NC) license \\ (https://creativecommons.org/licenses/by-nc/4.0/). See http://ivyspring.com/terms for full terms and conditions.
}

Received: 2018.03.26; Accepted: 2018.09.18; Published: 2018.10.20

\begin{abstract}
Introduction: Locally implanted antibiotic-eluting carriers may be a valuable adjuvant to the management of prosthetic joint infections. Aim: to assess local and plasma antibiotic concentrations as well as cumulative antibiotic urine excretion associated with clinical use of a gentamicin - or vancomycin-loaded mineral composite antibiotic carrier.

Methods: 32 patients (male/female $=19 / 13$, mean age $=56 ; 21-82$ years) were prospectively followed after implantation of gentamicin $(n=11)$, vancomycin $(n=15)$, or a combination $(n=7)$, using an antibiotic carrier (CERAMENT TM $\mid \mathrm{G}$ or CERAMENT ${ }^{\mathrm{TM}} \mid \mathrm{V}$, mean amount 11 (3-20) $\mathrm{mL}$ ) during resection arthroplasty of the hip/knee. We measured antibiotic concentrations in plasma $(1 \mathrm{~h}, 3 \mathrm{~h}$, $24 \mathrm{~h}, 48 \mathrm{~h}$ and $72 \mathrm{~h}$ post-implantation), urine $(24 \mathrm{~h}, 48 \mathrm{~h}$ and $72 \mathrm{~h}$ post-implantation) and in drain $(\mathrm{n}=15)$.

Results: We observed low antibiotic concentrations in plasma (Gentamicin: $0.33 \mathrm{mg} / \mathrm{L}(95 \%-\mathrm{Cl}$ : $0.25-0.44)$ and vancomycin: $1.33 \mathrm{mg} / \mathrm{L}(95 \%-\mathrm{Cl}: 1.02-1.66))$ and high concentrations in drain (Gentamicin: mean $57.8 \mathrm{mg} / \mathrm{L}$ (95\%-Cl: 45.8-69.7) and vancomycin: mean $234.4 \mathrm{mg} / \mathrm{L}$ (95\%-Cl: 198.9-269.7)). Use of a drain was associated with a statistically significant reduction in vancomycin urine excretion (55.6\% (95\% Cl: 36.45-74.92) to 28.71\% (95\% Cl: 13.07-44.35), $\mathrm{p}=0.042)$. A similar trend was observed for gentamicin (34.17\% (95\% Cl: $24.62-43.72)$ to $16.22 \%$ (95\% Cl: $0-33.86)$, $\mathrm{p}=0.078$ ).

Conclusions: CERAMENT ${ }^{\mathrm{TM}} \mathrm{G} / \mathrm{V}$ was associated with safe plasma concentrations and high local concentrations above minimum inhibitory concentration. Installation of a surgical drain results in removal of a substantial amount of antibiotics and reduces antibiotic urine excretion.
\end{abstract}

Key words: Antibiotic Carrier, Antibiotic Elution, Pharmacokinetics, Prosthetic Joint Infection

\section{Introduction}

The clinical and economic burden of implant-associated infections is projected to rise significantly during the coming decades (1), and rates of PJI (prosthetic joint infections) are especially increasing in patients with bone tumours, due to improved overall survival $(2,3)$. Immunosuppression, generally reduced performance status, and poor physiological reserve make the treatment of PJI in this patient population extremely challenging and demanding. Prosthetic implants remain particularly susceptible to infection throughout a patient's entire lifetime due to their role as a foreign body, leading to a persistent focal decrease in minimal infecting pathogen doses (4). Subsequent biofilm formation on 
the implant surface results in significantly increased tolerance to antibiotics, and demands antibiotics in extremely high concentrations if eradication of the respective microorganisms is to be achieved (5). After systemic administration, antibiotics must overcome absorption, protein-binding, ionisation and tissue diffusion. The rate and extent of aminoglycosides and glycopeptides into bone after systemic administration has been reported as bone: serum concentrations of 0.1 - 0.3. Accordingly, systemic administration of aminoglycosides and glycopeptides in the treatment of PJI may demand high serum concentrations (6). Furthermore, administrable amounts are limited by the risk of various toxic reactions (7). Local antibiotic delivery with an appropriate carrier can bypass these obstacles and achieve extremely high local concentrations of antibiotics, which are often necessary to eradicate or prevent PJI (8). Several methods for antibiotic delivery into an area of bone/prosthetic joint infection exist. The antibiotic carrier most frequently employed in clinical practice is PMMA: polymethyl-methacrylate, applied as beads, rods or spacers, and successful results have been reported for treatment of both osteomyelitis and PJI (9, 10). However, since PMMA is not biodegradable and continues to release antibiotics at low concentrations for many years, its use is associated with a need for secondary surgery (for removal of the PMMA), development of bacterial resistance, and possible re-colonisation $(8,11,12)$. There is an increasing role for alternative antibiotic-eluting carriers that are isothermic, provide high local concentrations of antibiotics (as well as sufficiently low corresponding plasma levels to avoid toxic damage), and are fully biodegradable to avoid bacterial resistance and re-colonisation. Until now, the concept of locally implanted antibiotics for biofilm-associated infections has been mostly studied in in-vitro models. However, their resemblance to in-vivo models and their clinical relevance are being questioned (13). In order to improve the understanding of the possible role of local antibiotic delivery in the treatment of chronic PJI, clinical data is imperative. The purpose of this study was to investigate clinical in-vivo antibiotic elution characteristics and pharmacokinetic properties of a commercially available antibiotic eluting BGS: Bone graft substitute that has shown promising in-vitro and in-vivo results in the treatment or prevention of bone and joint-infections $(8,11,14)$. In particular, we studied the local antibiotic concentrations achieved in-situ, the maximum antibiotic serum concentrations, and cumulative antibiotic excretion in urine over the first three post-operative days. An aminoglycoside (gentamicin) and/or a glycopeptide (vancomycin) were applied in a commercially available antibiotic-eluting BGS (bone graft substitute), for prevention or treatment of PJI. Gentamicin is a bacteriocidic agent that attacks both the ribosome and cell wall of the primarily gram-negative rod in aerobic conditions. Vancomycin attacks gram-positive cell walls, thereby working bactericidically (15). This is the first clinical study that investigates the in-vivo elution profile of this antibiotic carrier in plasma, drain-fluid and urine in a cohort of patients.

\section{Methods}

We prospectively evaluated 32 patients $(\mathrm{M}: \mathrm{F}=$ 19:13, mean age $=56$ (range 21-82) years), who underwent local implantation of a commercially available antibiotic-eluting BGS for prevention or treatment of PJI in our department between February 2016 and February 2017. 12 of cases were treated with therapeutic intent, with either DAIR (debridement, antibiotic and implant retention), or 1-stage or 2-stage revision for an acute or chronic PJI. 20 cases were treated with prophylactic intent during primary or revision implantation of a mega-implant, after either extensive primary bone tumour resection or revision of a loose and/or failed revision hip or knee arthroplasty (Table 1). The BGS utilised (CERAMENT $^{\mathrm{TM}}$, Bonesupport AB Lund, Sweden), is a bio-composite of calcium sulphate and hydroxyapatite, containing either $17.5 \mathrm{mg} / \mathrm{mL}$ of gentamicin (CERAMENT ${ }^{\mathrm{TM}} \mid \mathrm{G}$ ) or $66 \mathrm{mg} / \mathrm{mL}$ of vancomycin (CERAMENTTM $\mid \mathrm{V}$ ), which forms an injectable, rapidly hardening paste. The antibiotic-loaded BGS was either applied directly onto the surface of the endoprosthetic implants as a paste, injected into periprosthetic bone defects, or deposited in immediate proximity of the exposed bone and/or endoprosthetic components as hardened beads/rods. CERAMENT $^{\mathrm{TM}} \mid \mathrm{G}$ was implanted in 11 cases (mean volume $12.1 \mathrm{~mL}$ (range: $3-20 \mathrm{~mL}$ )), CERAMENT ${ }^{\mathrm{TM}} \mid \mathrm{V}$ in 15 cases (mean volume $11.1 \mathrm{~mL}$ (range: $5-20 \mathrm{~mL}$ ) and a combination of both products in the remaining 7 cases (mean volume of $9.7 \mathrm{~mL}$ (range $8-10 \mathrm{~mL}$ ) and $10 \mathrm{~mL}$, respectively) (Table 1 ). The choice between CERAMENTTM $^{\mathrm{TM}} \mid \mathrm{G}$, CERAMENT ${ }^{\mathrm{TM}} \mid \mathrm{V}$ or a combination of the two was made in collaboration between the microbiologist and surgeon based on the previously identified profile of pathogens. We analysed drain fluid, serum, and urine sample concentrations of gentamicin and vancomycin to assess in-vivo elution characteristics and pharmacokinetic behaviour of these two antibiotics after release from the carrier. Patients receiving concomitant systemic gentamicin or vancomycin were not included in the study. Blood was collected after (A) 30 minutes, (B) 3 hours, (C) 24 hours, (D) 48 hours, 
and (E) 72 hours post-implantation. Samples were centrifuged at 3000 RPM: Rounds per minute, (1500G) for 10 minutes. The supernatant was transferred to a $2 \mathrm{~mL}$ polypropylene tube and placed in a bio-freezer at $-80^{\circ} \mathrm{C}$ for subsequent antibiotic concentration measurements. In 15 patients, a deep surgical wound drain was applied. In these patients, total drain output volume was measured when the drain was removed after a mean of 90 hours (range: 48-144, removal criteria: $<50 \mathrm{~mL}$ drain fluid production over the previous $24 \mathrm{~h}$ ). Drain fluid was collected at a single point in time (when the drain was removed) to obtain data on the total cumulative loss of antibiotic through the surgical drain. Drain fluid was transferred to a $5 \mathrm{~mL}$ heparin tube, centrifuged at 3000RPM (1500G) for 10 minutes, and the supernatant transferred to a $2 \mathrm{~mL}$ polypropylene tube and placed in a bio-freezer at $-80^{\circ} \mathrm{C}$ prior to concentration analysis. 24-hour urine output was collected over three days, counting from the time of antibiotic implantation $(t=0)$. The volume was registered for each $24 \mathrm{~h}$ urine container, and samples were taken separately and immediately transferred to a bio-freezer at $-80^{\circ} \mathrm{C}$ in a $4 \mathrm{~mL}$ polypropylene tube for subsequent antibiotic concentration analysis. Antibiotic concentrations were analysed using MULTIGEN; a PETINA (particleenhanced turbid metric inhibition immunoassay) in all three media.

\section{Statistics and ethical approval}

We performed an unpaired t-test to compare the cumulative antibiotic urine output between patients with deep wound drain and no deep wound drain, respectively. P-values $<0.05$ were considered statistically significant. SPSS version 23 and SAS Enterprise 7.1 software were used for statistical assessments. Data is presented as mean and with 95\% confidence intervals $(95 \% \mathrm{CI})$. The study has been approved by the National Data Protection Agency (J.no.2013-41.2591) and regional ethics committee (J. no H-3-2014-133). All procedures were in accordance with the ethical standards of the responsible committee on human experimentation and with the Helsinki Declaration of 1975, as revised in 1983.

\section{Results}

We obtained an average cumulative drain output of $698 \mathrm{~mL}$ (range: $50-3500 \mathrm{~mL}$ ), which was collected over a mean of $90 \mathrm{~h}$ (range: 48-144) post-antibiotic implantation (Table 2), with mean drain-fluid concentrations of $57.8 \mathrm{mg} / \mathrm{L}$ (95\% CI: 45.8-69.7) for gentamicin and $234.4 \mathrm{mg} / \mathrm{L}$ (95\% CI: 198.9-269.7) for vancomycin, respectively. We found a mean plasma gentamicin concentration of $0.33 \mathrm{mg} / \mathrm{L}(95 \% \mathrm{CI}$ : $0.25-0.44)$ and a mean plasma vancomycin concentration of $1.33 \mathrm{mg} / \mathrm{L}$ (95\% CI: 1.02-1.66). The in-vivo plasma concentration profile of gentamicin peaked very early after antibiotic implantation $(3 \mathrm{~h}$ post-op), followed by a fast and persistent drop during the remaining period of analysis, whereas the respective vancomycin plasma concentration profile peaked somewhat later ( $24 \mathrm{~h}$ post-op) and tended to drop slower (figure. 1). The maximum individual concentration measured was $2.14 \mathrm{mg} / \mathrm{L}$ for gentamicin and $10.32 \mathrm{mg} / \mathrm{L}$ for vancomycin. A comparison to the trough toxic levels of gentamicin $(2 \mathrm{mg} / \mathrm{L})$ and vancomycin $(10 \mathrm{mg} / \mathrm{L})(11,16)$ is illustrated in figure. 2. We did not observe any alteration in the elution profile in cases where gentamicin and vancomycin were combined.

Table 1. Treatment characteristics in 32 cases with locally implanted Gentamicin and/or Vancomycin in a commercially available antibiotic carrier (CERAMENT TM)

\begin{tabular}{|c|c|c|c|c|}
\hline & Gentamicin & Vancomycin & \multicolumn{2}{|l|}{ Combined } \\
\hline Gender (M:F) & $7: 4$ & $7: 7$ & \multicolumn{2}{|l|}{$5: 2$} \\
\hline \multicolumn{5}{|l|}{ Indication \& Procedure } \\
\hline Therapeutic (PJI) & 3 & 6 & \multicolumn{2}{|l|}{3} \\
\hline DAIR & 1 & 2 & \multicolumn{2}{|l|}{2} \\
\hline 1-Stage & 2 & 2 & \multicolumn{2}{|l|}{0} \\
\hline 2-Stage & 0 & 2 & \multicolumn{2}{|l|}{1} \\
\hline Prophylactic & 8 & 8 & \multicolumn{2}{|l|}{4} \\
\hline Primary Tumour-prosthesis & 7 & 4 & \multicolumn{2}{|l|}{3} \\
\hline Revision Arthroplasty & 1 & 4 & \multicolumn{2}{|l|}{1} \\
\hline \multirow{3}{*}{$\begin{array}{l}\text { Antibiotic Carrier } \\
\text { mean volume }(\mathrm{mL}) \text { and corresponding antibiotic } \\
\text { concentration }(\mathrm{mg}) \text {. }\end{array}$} & & & \multicolumn{2}{|c|}{ CERAMENT $^{\mathrm{TM}} \mid \mathrm{G} \&$ CERAMENT ${ }^{\mathrm{TM}} \mid \mathrm{V}$} \\
\hline & $12.1(3-20)$ & $11.1(5-20)$ & $9.7(8-10)$ & $10(10-10)$ \\
\hline & $211,75(52,5-350)$ & $732,6(330-1320)$ & $169,75(140-175)$ & $660(660-660)$ \\
\hline
\end{tabular}


A)

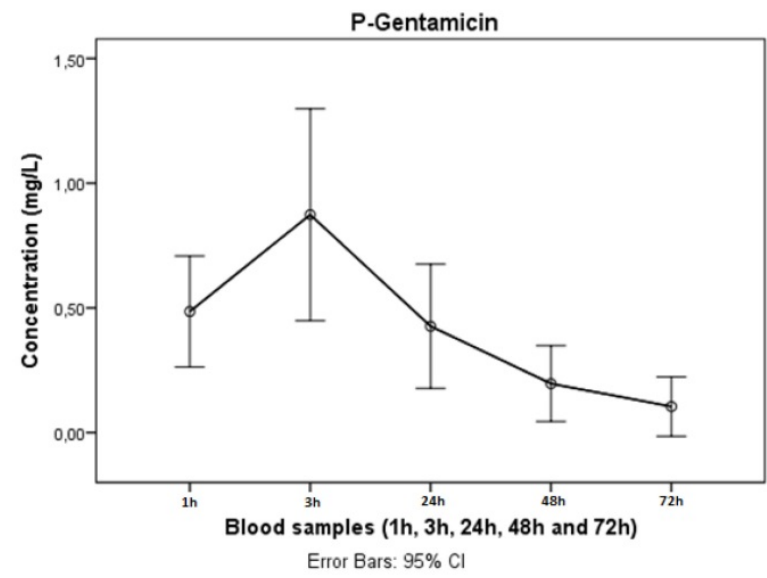

B)

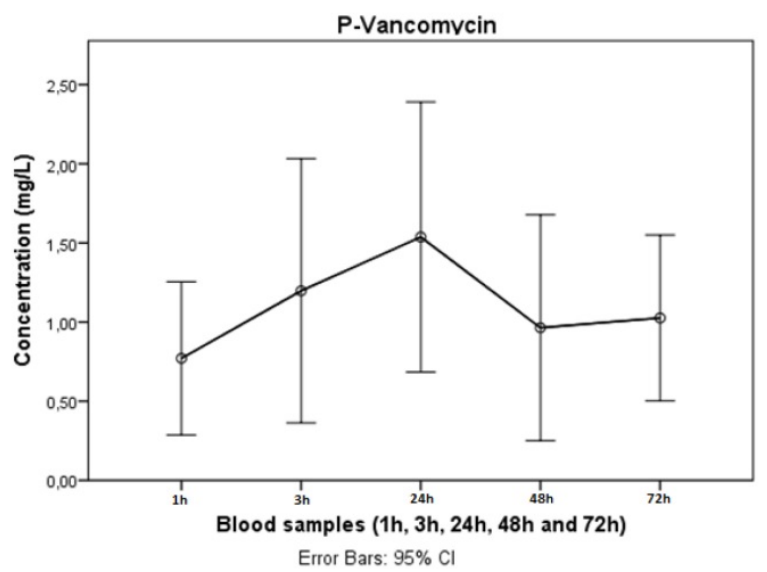

Figure 1. Mean plasma-concentration of $(A)$ gentamicin and $(B)$ vancomycin during the first three days post antibiotic implant. Time points indicate 1, 3, 24, 48 and 72 hours post-operatively. Error bars indicate standard error of mean.

Table 2. Gentamicin and vancomycin concentrations in drain fluid, plasma and urine in 32 cases treated with a commercially available antibiotic carrier (CERAMENT TM $)$

\begin{tabular}{llllll}
\hline & Gentamicin & & \multicolumn{2}{l}{ Vancomycin } \\
\hline & Cases (n) & Mean concentration (mg/L) & $95 \%$ CI & Cases (n) & Mean Concentration (mg/L) 95\% CI \\
Drain & 5 & 57.8 & $45.8-69.7$ & 11 & 234.4 \\
Plasma (peak) & 17 & $0.33(2.14)$ & $0.25-0.44$ & 15 & $1.33(10.32)$ \\
Urine (No drain) & 12 & 23.32 & $14.0-32.6$ & 10 & $198.9-269.7$ \\
Urine (Drain) & 5 & 10.59 & $0-25.6$ & $1.02-1.66$ & 121.02 \\
\hline
\end{tabular}

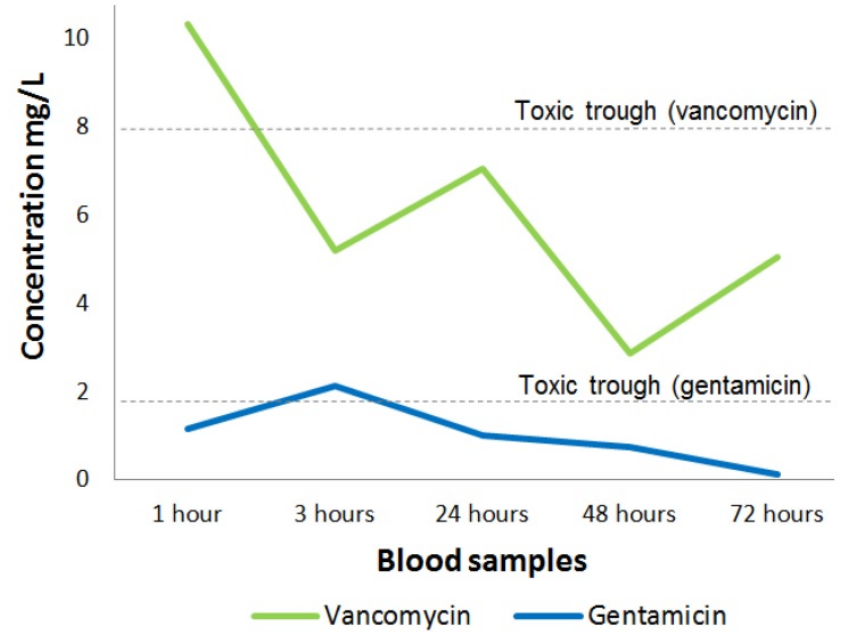

Figure 2. Peak plasma-concentrations (highest measured antibiotic concentration) of gentamicin (blue) and vancomycin (green) during the first three days post antibiotic implant with the corresponding toxic trough levels.

The mean percentage of locally implanted gentamicin, excreted in urine after 3 days, was $16.22 \%$ (95\% CI: 0-33.86) and 34.17\% (95\% CI: 24.62-43.72) for cases with $(n=5)$ or without $(n=12)$ a surgical drain. $100 \%$ refers to the total amount of implanted antibiotics. For vancomycin, the corresponding ratios were $28.71 \%$ (95\% CI: 13.07-44.35) and 55.6\% (95\% CI: $36.45-74.92)$ for patients with $(n=11)$ or without $(n=10)$ a surgical drain, indicating that the installation of a deep wound drain substantially decreases cumulative antibiotic urine excretion (table 2 presented as $\mathrm{mg} / \mathrm{L}$ and figure 3 presented as cumulative percentages). In patients treated with local vancomycin, the observed difference in excretion was statistically significant $(p=0.042)$. In patients treated with gentamicin, a similar tendency was observed but did not quite reach statistical significance $(p=0.078)$.

\section{Discussion}

\section{Drain fluid - local concentration}

Compared to the conventional minimally inhibitory concentrations (MIC) reported for planktonic pathogens typically involved in PJI (17, 18), the drain fluid concentrations we found in our study were approximately 30-60 times higher for gentamicin and vancomycin, respectively. Local antibiotic concentrations at these levels have been shown to eradicate planktonic bacteria and prevent implant surface colonisation in-vitro (8), which may potentially translate to a risk reduction for PJI. It has been reported, however, that mature biofilm requires significantly higher threshold-levels and MBEC (minimal biofilm eradicating concentrations) - over 1000 times greater than the corresponding MIC (18, 19). Together with other researchers in a previous study investigating the same antibiotic carrier in-vivo, 
A)

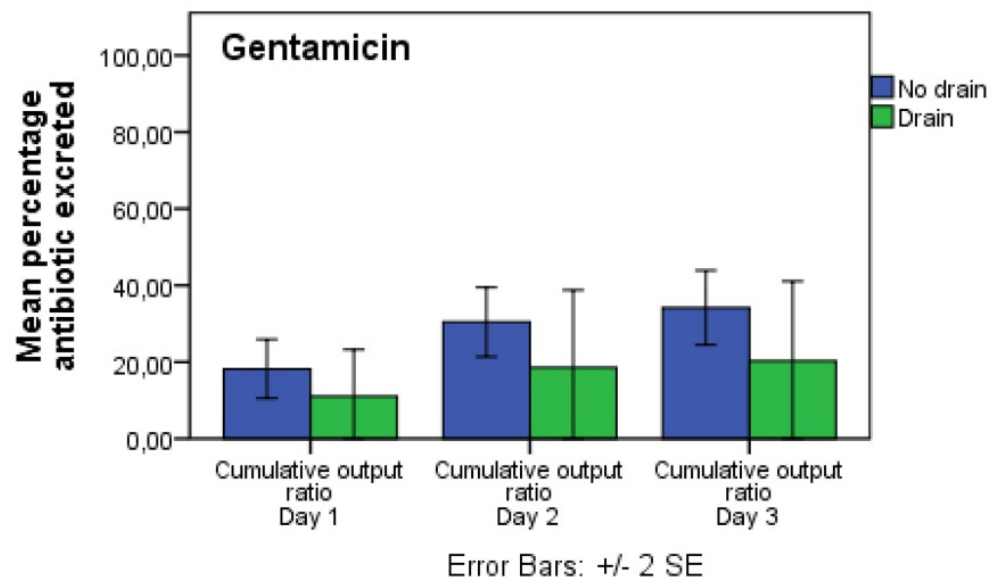

B)

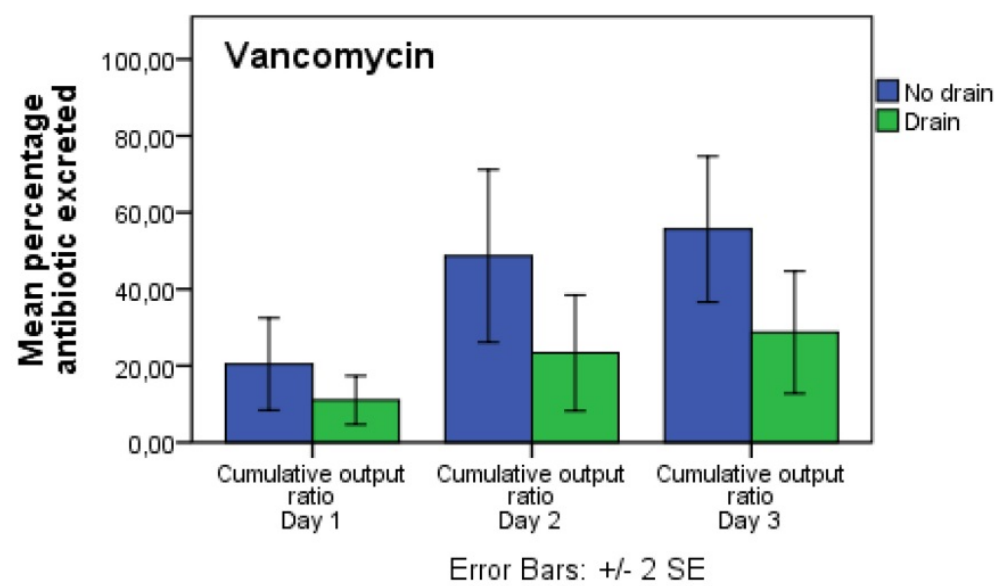

Figure 3. Cumulative urine output of $(A)$ gentamicin and $(B)$ vancomycin. Blue bars indicate patients without a surgical drain and green bars indicate patients with a surgical drain. Error bars indicate standard error of mean.

we found local gentamicin concentrations of up to $2000 \mathrm{mg} / \mathrm{L}$, indicating that antibiotic concentrations significantly above MBEC levels are achievable using the tested material as a local antibiotic carrier (11). Due to differences in study designs, we were unable to reproduce such high concentrations in our drain-fluid samples in this study. For reasons of simplicity, we used the antibiotic concentration in drain fluid as a surrogate measure of the in-vivo local concentration, because measurement of actual local antibiotic concentrations is difficult and not feasible without an additional intervention in-vivo in a clinical context. It is possible that actual local antibiotic concentrations and corresponding potential bacteria-eradicating ability, are higher in areas of proximity to, or within the antibiotic-loaded carrier or lower in more peripheral wound compartments at greater distance or even separated from the carrier. Additionally, although it can be considered an important supplement in the understanding of antibiotic effect, the antibiotic concentration analysed in a particular compartment is not a unique measure of the antibiotic activity and potential pathogen eradication. This measurement needs to be considered within the overall clinical scenario (17). To our knowledge, clinical results of local use of antibiotic-eluting BGS for PJI have, so far, only been published in case reports $(20,21)$. However, excellent clinical results have been reported in related scenarios of biofilm-associated bone and joint infections. McNally et. al. achieved eradication of infection in a cohort of 100 patients with chronic osteomyelitis, treated with a single-stage protocol and dead-space management with the same antibiotic carrier (14), and concluded that the surgical treatment facilitated by the antibiotic carrier was an effective and patient-friendly treatment of chronic osteomyelitis.

\section{Plasma - systemic toxicity}

It is well known that elevated systemic levels of gentamicin and vancomycin may cause toxic ear damage and renal failure, and antibiotic trough levels must therefore be carefully monitored to guide the dosage of the antibiotic to minimise the risk of complications. Trough toxic levels are estimated to be $2 \mathrm{mg} / \mathrm{L}$ for gentamicin and $10 \mathrm{mg} / \mathrm{L}$ for vancomycin $(11,16)$. Accordingly, the plasma levels observed in this study were well below these levels and cannot be considered nephrotoxic or ototoxic. This finding agrees with other studies examining the same antibiotic carrier, confirming equally low plasma levels and/or no side-effects related to antibiotic toxicity $(11,22,23)$. However, other studies report potential toxic antibiotic levels when using local antibiotic eluting grafts (24) and special caution must be taken when a patient is suffering with concomitant renal failure (25). A potential risk of antibiotic toxicity must, therefore, still not be neglected, particularly when using local antibiotics in extreme dosages, regardless of the carrier material through which they are applied. Finally, in order to compare this data appropriately with that reported on PMMA, in addition to serum plasma-concentration measurements, thorough clinical follow-up with regards to possible side-effects is required for up to 
five years post-implantation, as reported in early PMMA in-vitro elution studies (12).

\section{Urine output - elution and excretion}

Measurement of antibiotic concentrations in urine is a simple and non-invasive method to indirectly monitor the elution from an antibiotic carrier in-vivo. A previous investigation of urine elution from various antibiotic carriers was able to trace gentamicin in urine for up to 95 days (10). The results observed in our study are comparable with a similar study that previously analysed urine sample concentrations after local implantation of the same antibiotic-loaded carrier (CERAMENT ${ }^{\mathrm{TM}} \mid \mathrm{G}$ ) for another set of indications, and found the same overall urine excretion profile with detectable levels for up to 7 days post-surgery (11). Neither of these two or any other previous studies, however, have investigated or quantified the effect of a deep drain on antibiotic urine output. In our study, pooled daily urine outputs were measured to study the potential amount of antibiotic absorbed from the implantation site and subsequently excreted in urine. Theoretically, $95 \%$ of vancomycin and $60-90 \%$ of gentamicin is excreted in the kidneys, unchanged when administered intravenously (15). However, since antibiotic absorption and elimination is dependent on the available amount of local antibiotic, our results clearly indicate that use of a deep drain that physically removes fluid, as well as antibiotics from the site of operation significantly affects the amount of antibiotic excreted in urine. Accordingly, it is likely that the amount of locally available antibiotic is equally reduced by one third to one half, which potentially compromises the desired antimicrobial effect of the locally implanted drug-eluting carrier. We therefore recommend avoiding use of deep surgical drains in conjunction with implantation of local antibiotic carriers, if possible.

In conclusion, our study shows that local implantation of a commercially available gentamicinand vancomycin-eluting BGS with the intention to prevent or treat PJI, is associated with high local concentrations, which by far exceed the MIC for most bacteria involved in prosthetic joint infections, and safe low plasma-concentrations for both antibiotics. Accordingly, CERAMENT ${ }^{\mathrm{TM}}$ might be a valuable addition to the treatment/prevention of PJI, however, use of a surgical drain may significantly reduce the amount of locally available antibiotic and should therefore be avoided if possible. Further clinical studies are needed to confirm whether this translates into a noticeable clinical effect. Based on our observations, antibiotic urine concentration measurements can be an effective, non-invasive method to monitor the in-vivo antibiotic elution from an antibiotic carrier in clinical practice.

\section{Acknowledgements}

Niels Frimodt Møller, Professor, DMSc, Chief of Department of Clinical Microbiology, Rigshopitalet, University Hospital of Copenhagen, Denmark.

\section{Funding}

This study was funded by BONESUPPORT AB.

\section{Author Contributions}

Thomas Colding-Rasmussen: Data collection and analysis, preparation of tables and figures, drafting of the manuscript.

Peter Horstmann: Data collection and analysis curation, preparation of tables and figures.

Michael Mørk Petersen: Validation of data analysis, review and editing of the manuscript, acquisition of funding.

Werner Hettwer: Conceptualisation, project administration and supervision, writing, review, and editing of the manuscript.

\section{Competing Interests}

The authors have declared that no competing interest exists.

\section{References}

1. Mauffrey C, Herbert B, Young H, Wilson ML, Hake M, Stahel PF. The role of biofilm on orthopaedic implants: the "Holy Grail" of post-traumatic infection management? Eur J Trauma Emerg Surg. 2016;42(4):411-6.

2. Colding-Rasmussen T, Thorn AP, Horstmann P, Rechnitzer C, Hjalgrim LL, Krarup-Hansen A, et al. Survival and prognostic factors at time of diagnosis in high-grade appendicular osteosarcoma: a 21 year single institution evaluation from east Denmark. Acta Oncol. 2017:1-6.

3. Mavrogenis AF, Papagelopoulos PJ, Coll-Mesa L, Pala E, Guerra G, Ruggieri P. Infected tumor prostheses. Orthopedics. 2011;34(12):991-8; quiz 9-1000.

4. Trampuz A, Zimmerli W. Prosthetic joint infections: update in diagnosis and treatment. Swiss Med Wkly. 2005;135(17-18):243-51.

5. de Oliveira A, Cataneli Pereira V, Pinheiro L, Moraes Riboli DF, Benini Martins K, Ribeiro de Souza da Cunha Mde L. Antimicrobial Resistance Profile of Planktonic and Biofilm Cells of Staphylococcus aureus and Coagulase-Negative Staphylococci. Int J Mol Sci. 2016;17(9).

6. Landersdorfer CB, Bulitta JB, Kinzig M, Holzgrabe U, Sorgel F. Penetration of antibacterials into bone: pharmacokinetic, pharmacodynamic and bioanalytical considerations. Clin Pharmacokinet. 2009;48(2):89-124.

7. Levison ME, Levison $\mathrm{JH}$. Pharmacokinetics and pharmacodynamics of antibacterial agents. Infect Dis Clin North Am. 2009;23(4):791-815, vii.

8. Butini ME, Cabric S, Trampuz A, Di Luca M. in vitro anti-biofilm activity of a biphasic gentamicin-loaded calcium sulfate/hydroxyapatite bone graft substitute. Colloids Surf B Biointerfaces. 2017;161:252-60.

9. Tande AJ, Patel R. Prosthetic joint infection. Clin Microbiol Rev. 2014;27(2):302-45.

10. Webb JC, Spencer RF. The role of polymethylmethacrylate bone cement in modern orthopaedic surgery. J Bone Joint Surg Br. 2007;89(7):851-7.

11. Stravinskas M, Horstmann P, Ferguson J, Hettwer W, Nilsson M, Tarasevicius $\mathrm{S}$, et al. Pharmacokinetics of gentamicin eluted from a regenerating bone graft substitute: in vitro and clinical release studies. Bone Joint Res. 2016;5(9):427-35.

12. Wahlig H, Dingeldein E. Antibiotics and bone cements. Experimental and clinical long-term observations. Acta Orthop Scand 1980:51(1):49-56.

13. Bjarnsholt $T$, Alhede $M$, Alhede M, Eickhardt-Sorensen SR, Moser C, Kuhl M, et al. The in vivo biofilm. Trends Microbiol. 2013;21(9):466-74.

14. McNally MA, Ferguson JY, Lau AC, Diefenbeck M, Scarborough M, Ramsden $\mathrm{AJ}$, et al. Single-stage treatment of chronic osteomyelitis with a new absorbable, gentamicin-loaded, calcium sulphate/hydroxyapatite biocomposite: a prospective series of 100 cases. Bone Joint J. 2016:98-B(9):1289-96 
15. [Internet] Jørgen E. Engberg NF-M. Medicin.dk, Aminoglykosider til systemisk brug2017 [Available from: http://pro.medicin.dk/ Laegemiddelgrupper/Grupper/193010\#a110.

16. James CW, Gurk-Turner C. Recommendations for monitoring serum vancomycin concentrations. Proc (Bayl Univ Med Cent). 2001;14(2):189-90.

17. Mouton JW, Theuretzbacher U, Craig WA, Tulkens PM, Derendorf H, Cars O. Tissue concentrations: do we ever learn? J Antimicrob Chemother. 2008;61(2):235-7.

18. Brady AJ, Laverty G, Gilpin DF, Kearney P, Tunney M. Antibiotic susceptibility of planktonic- and biofilm-grown staphylococci isolated from implant-associated infections: should MBEC and nature of biofilm formation replace MIC? J Med Microbiol. 2017;66(4):461-9.

19. Sandoe JA, Wysome J, West AP, Heritage J, Wilcox MH. Measurement of ampicillin, vancomycin, linezolid and gentamicin activity against enterococcal biofilms. J Antimicrob Chemother. 2006;57(4):767-70.

20. C. Jensen WH, P. Horstmann, M. Petersen. C. Jensen, W. Hettwer, P. Horstmann, M. Petersen. 34th EBJIS 2015 online abstract P25 - (\#210)2015.

21. Peter Frederik Horstmann WH, Michael, Petersen M. USE OF A VANCOMYCIN-ELUTING BONE GRAFT SUBSTITUTE FOR LOCAL ANTIBIOTIC DELIVERY IN ONE-STAGE REVISION OF AN INFECTED TOTAL FEMORAL REPLACEMENT - A CASE REPORT. 35th EBJIS 2016 online abstract P602016.

22. Ferguson J, Diefenbeck M, McNally M. Ceramic Biocomposites as Biodegradable Antibiotic Carriers in the Treatment of Bone Infections. J Bone Jt Infect. 2017;2(1):38-51.

23. Logoluso N, Drago L, Gallazzi E, George DA, Morelli I, Romano CL. Calcium-Based, Antibiotic-Loaded Bone Substitute as an Implant Coating: A Pilot Clinical Study. J Bone Jt Infect. 2016;1:59-64.

24. Swieringa AJ, Tulp NJ. Toxic serum gentamicin levels after the use of gentamicin-loaded sponges in infected total hip arthroplasty. Acta Orthop. 2005;76(1):75-7.

25. James A, Larson T. Acute renal failure after high-dose antibiotic bone cement: case report and review of the literature. Ren Fail. 2015;37(6):1061-6. 